\title{
Headache and Central Positioning Vertigo in a Middle Aged Female-a Case of Solitary Cerebellar Tuberculoma Involving Left Cerebellar Hemisphere
}

\author{
Shakya Bhattacharjee, Arijit Majumdar*, Angshuman Jana** \\ Neurology Itu, Institute of Neurosciences, Kolkata,India \\ *SSKM Hospital Department of Pathology, Kolkata, India \\ **SSKM Hospital Department of Microbiology, Kolkata, India
}

\begin{abstract}
Summary
A 48-yr-old female presented with headache and an illusory sensation of spinning of head in respect to environment for last 8 weeks. Her head spinning or vertigo had no particular direction or not precipitated by any specific head posture. Headache is non- specific in nature and intensified in last few days. Her neurological examination revealed a central positional vertigo with horizontal gaze evoked nystagmus and ataxia. Her MRI scan brain showed the presence of a large solitary ring enhancing lesion in the left cerebellar hemisphere. The lesion was surgically excised and was examined histopathologically that revealed a chronic inflammatory granuloma with caseation necrosis and multinucleated giant cells suggestive of tuberculosis. (Turkish Journal of Neurology 2012; 18:39-42)
\end{abstract}

Key Words: Headache, caseous necrosis, cerebellum, tuberculoma

\section{Introduction}

Tuberculomas are avascular, spherical, granulomatous masses of small tubecles (1). The incidence of tuberculoma in different parts of the world is different. As is the case with tuberculosis in general there has been a significant decline in the incidence of tuberculoma worldwide. Though solitary tuberculoma is seen occasionally but multiple grape like tuberculoma are rare which develop as a result of coalescence of multiple small immature tuberculoma and resemble a cluster of neurocysticercus cyst (1).

\section{Case Report}

A 48-yr-old apparently healthy woman presented with a complaint of progressive headache, feeling of spinning around the head over last 8 weeks. On detailed enquiry, she stated that initially she had an illusory feeling of spinning of the head in respect to the surroundings. The spinning sensation of the head was mild, almost persistent in nature having no particular direction of spinning and not precipitated by any particular head position. She has no complaint suggestive of seizure, diplopia, tinnitus or impairment of hearing although she sometimes tends to tilt towards left side while walking. Later she developed non-specific headache mostly on the occipital region especially when leaning forward. There was no other specific aggravating or relieving factors for the headache. Though initially the headache was relieved during sleep and simple analgesics but for the last 10 days or so the headache is disrupting her sleep and persists even during the daytime necessitating frequent use of non steroidal analgesics but temporary relief only. The headache was not associated with dimness of vision, loss of consciousness, palpitation, light headedness, syncope or near syncopal attack, convulsion, loss of body weight, fever or loss of appetite although occasional nausea and vomiting were there. She has no history of diabetes mellitus, hypertension, tuberculosis, heart disease or any other major illness. Neither had she any history of similar episode in the past.

Address for Correspondence/Yazıșma Adresi: Shakya Bhattacharjee MD, Neurology Itu, Institute of Neurosciences, Kolkata, India 
Her cardiological, respiratory, gastrointestinal examinations revealed no obvious abnormality. Her vital signs were also stable. A detailed eye examination including the ophthalmoscopy revealed no suggestive abnormality.

Dix-Hallpike maneuver triggered mild vertigo and nystagmus immediately with no features of fatigability or lessening of the symptoms with repeated trials/maneuvers. On clinical examination, no cranial nerve abnormality including extra-ocular muscle paresis was detected. There was gaze evoked jerky horizontal nystagmus with direction of the fast component to the left but no overshoot of target directed fast eye movements (resembling past pointing of the limbs) or hypometric saccades. No vertical gaze movement restriction, vertical gaze nystagmus or burst of consecutive saccades (opsoclonus) noted during clinical examination. Finger nose finger test of the left hand was abnormal and the patient demonstrating truncal ataxia and wide based gait but she did not reveal other signs of cerebellar involvement like scanning speech, dysdiadochinesia, pendular knee jerk. Her Romberg's test was negative.

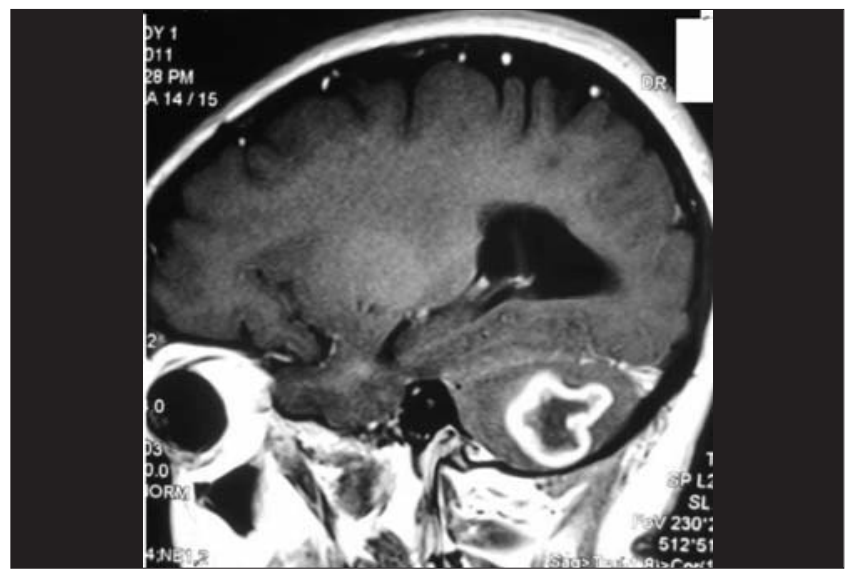

Figure 1. Sagittal section of T1 weighted MRI with contrast shows ring enhancing lesion in the cerebellum.

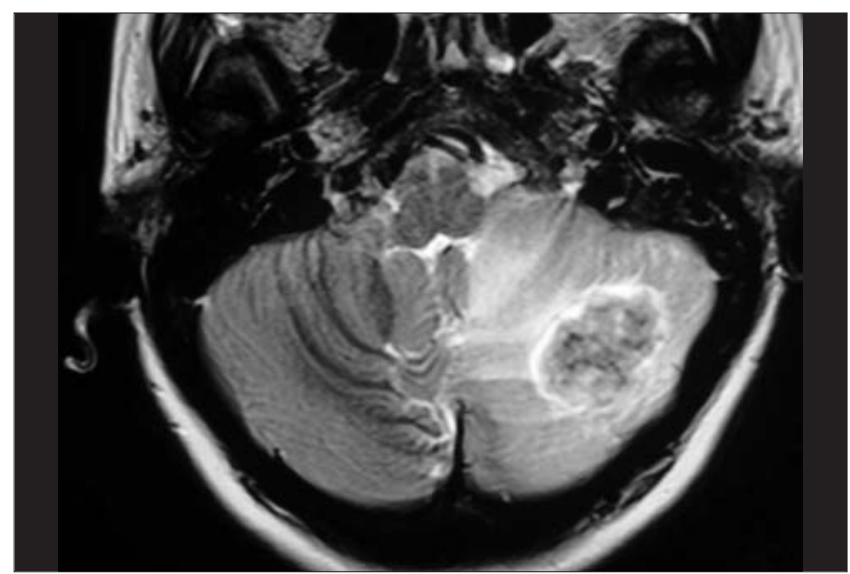

Figure 2. Axial T2-weighted view of the MRI showing the lesion in the cerebellum.
Her complete blood count revealed mild anemia and ESR $45 \mathrm{~mm}$ in the 1 st hour. Her liver function test, renal functions and electrolytes were within normal limits. Mantoux test was strongly positive though she is sputum negative on 3 consecutive morning samples. Her X-ray chest PA view showed no obvious abnormality. Antibodies to HIV 1 and 2, and VDRL were not detected; IgG and IgM antibodies against tuberculosis were raised. CT scan abdomen and thorax with contrast could not detect any obvious abnormality including any enlarged lymph nodes. ECG, Treadmill test, echocardiography were also normal.

Contrast MRI scan of brain revealed a solitary ring enhancing lesions in the left cerebellar hemisphere with mild obstructive hydrocephalus above the 4th ventricle (Figure 1,2). A retro mastoid suboccipital craniotomy was performed and gross excision of the cerebellar Space-Occupying-Lesion (SOL) was done (Figure 3). The material was sent for histopathological examination.

Considering the sub-acute onset with no obvious source of any malignancy or other granulomatous disease to justify the origin of the ring enhancing lesions in the CT/MRI and endemicity of tuberculosis in our country (India) she was diagnosed provisionally as a case cerebellar tuberculoma. Later histopathological examination of the cerebellar SOL showed

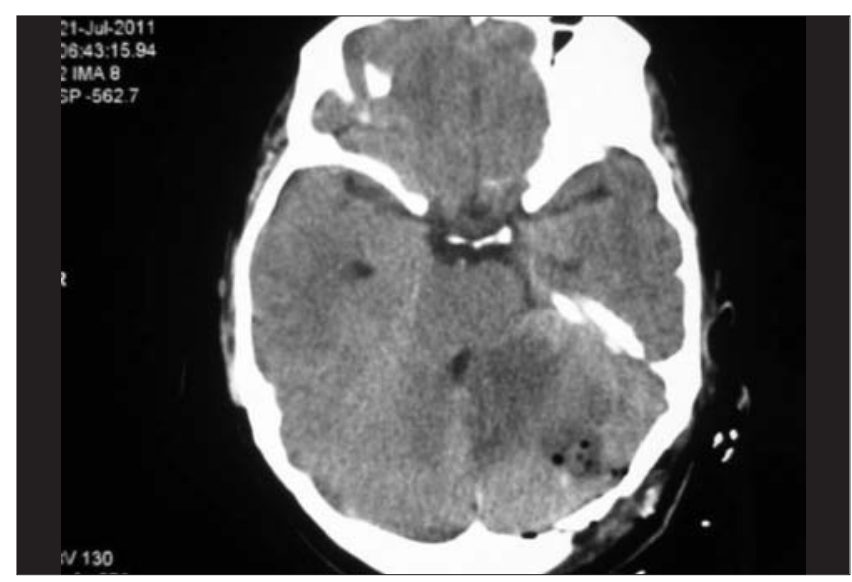

Figure 3. CT brain with contrast following the retro mastoid suboccipital craniotomy with gross excision of the cerebellar SOL.

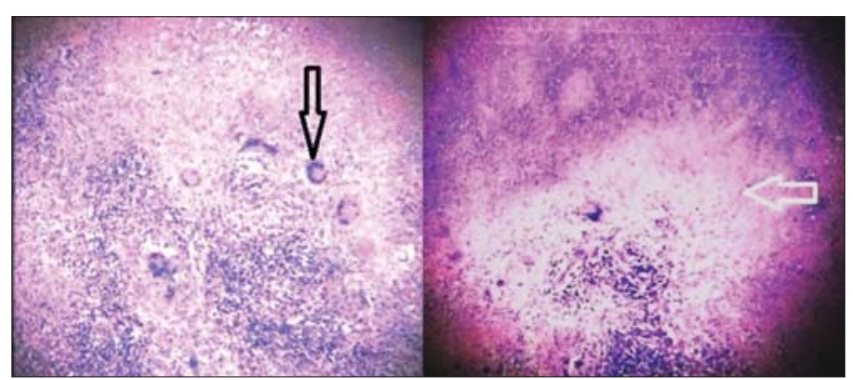

Figure 4. Histopathological examination showing caseous necrosis (white arrow) and multinucleated Langhans' giant cell (black arrow) suggestive of tubercular granuloma. 
caseating granuloma (white arrow) and multinucleated Langhans' type of giant cells suggestive of tuberculoma of the left cerebellar hemisphere. Here in the Langhans' giant cells the nuclei are arranged in horseshoe shaped fashion along the periphery (Figure 4, black arrow).

Anti tubercular drugs were administered in the following dose: Isoniazid $300 \mathrm{mg} /$ day, rifampicin $450 \mathrm{mg} /$ day, pyrazinamide $750 \mathrm{mg}$ twice a day, ethambutol $1200 \mathrm{mg} /$ day and pyridoxine $40 \mathrm{mg} /$ day. The drugs are being continued along with regular follow up clinically and also with investigations like liver function, complete blood count, uric acid and fundoscopic examinations.

\section{Discussion}

The differential diagnoses of solitary ring enhancing lesions are wide. Important considerations in our country (India) are tuberculoma, neurocysticercosis, metastatic deposits, lymphomas, primary brain tumors like glioma, toxoplasmosis, arterio-venous malformations (AVM), and vasculitis $(1,2)$. Sometimes, a giant tuberculoma very strongly mimicks a malignant tumor radiologically, creating great deal of confusion (3). In certain parts of USA fungal granulomas (histoplasmosis) are also to be kept in mind besides other granulomatous diseases like sarcoidosis, Wegener's granulomatosis, etc.

It is unlikely for a neurocysticercosis ring to have more than $20 \mathrm{~mm}$ size, midline shift, irregular thick outline $(>20 \mathrm{~mm})$ and an absence of scolex in MRI (4-6). Caseating granuloma like tuberculoma with a solid centre are hypointense or isointense on both $\mathrm{T} 1$ and $\mathrm{T} 2 \mathrm{~W} 1$ and show ring enhancement. Those with liquid centre are hypointense on T1W1, the centre is hyperintense on T2W1 but the periphery is hypointense and shows ring enhancement (1). Lipids within the macrophage are the major source of low signal T2W1 in tuberculoma $(1,7)$. Another close differential diagnosis is metastasis but in this case the histopathological examination showed no evidence of malignancy.

Central positional vertigo demonstrates mild vertigo and nystagmus immediately with no features of fatigability or lessening of the symptoms even with repeated Dix-Hallpike maneuver. Cerebellar lesion and other posterior fossa lesions constitute an important cause of central positioning vertigo.

Constitutional symptoms are uncommon and the patient is generally well. Focal seizures are the most common symptoms. Headache in this patient resulted from focal irritation or displacement of the pain sensitive structures or from the raised ICT. In fact, a headache that worsens rather than abates with recumbency is suggestive of a mass lesion (8). Multiple and large lesions may be associated with features of raised intracranial tension. Infratentorial tuberculomas may present with brainstem syndromes, cerebellar signs and multiple lower cranial nerve palsies apart from obstructive hydrocephalus (1). Concomitant extracranial tuberculosis is present in $30-50 \%$ cases but tuberculoma is not often associated with tubercular meningitis. Microtuberculoma (very small ones) probably represents the initial stages of the evolution of a tuberculoma. Calcification of a tuberculoma is uncommon and happens only in $2-6 \%$ of cases (1). It is noteworthy that not all of the calcified lesions are inactive or healed. Sometimes large tuberculoma can have broad dural attachments with feeding meningeal arteries leading to the lesion masquerading as meningioma $(1,9)$.

Most of the cases of intracranial tuberculoma are seen below the age 20 years. Infra-tentorial tuberculomas are more common in children, whereas supra-tentorial lesions are commoner in adults. About 15-20\% of patients have multiple tuberculomas. Cerebellar involvement in central nervous system tuberculoma is rare. In a series of 23 central nervous system tuberculoma cases Bayindir et al. could detect only 2 cases of cerebellar involvement (10).

Although definite diagnosis of brain tuberculoma needs histopathological confirmation of a brain biopsy specimen $(11,12)$ (not a practical approach in all cases), many neurologists are of the opinion that suggestive CT/MRI ring enhancing lesions in the brain with apparently no major symptoms in an ambulatory patient from endemic areas strongly justify the initiation of antitubercular drugs even if histopathological lesion is not feasible $(1,12)$.

In this case the histopathological examination following excision pinpointed the diagnosis of tuberculoma although not all intracranial SOL are amenable to surgery so often a ring enhancing lesion is treated presumptively based on the clinical features, other investigations, anatomical and geographical location. In fact a Langhans' giant cell mostly limits the differential diagnosis between tuberculosis and sarcoidosis but sarcoidosis never shows caseation in the granuloma (13). A zone of gliosis usually surrounds the area of the tuberculoma and advanced cases may show foci of calcification.

\section{References}

1. Ahmad FU, Gupta A, Mehta VS. In: VK Arora (ed). Practical Approach to Tuberculosis Management. 1st ed. New Delhi: Jaypee brothers (Medical Publishers Pvt Ltd.), 2006;138-48.

2. Garcia HH, Gonzalez AE, Evans CAW, et al. Taenia solium cysticercosis. Lancet 2003;361;547-56.

3. Poonnoose SI, Singh S, Rajsekhar V. Giant cerebellar tuberculoma mimicking a malignant tumor. Neuroradiology 2004;46:136-9.

4. VVeena Kalra. In: A Parthasarathy PSN Menon et al (eds). IAP Textbook of Pediatrics. 4 th ed. New Delhi: Jaypee Brothers Medical Publishers(P) Ltd, 2009;469-70. 
5. Rajshekhar V, Haran P, Prakash SG, Chandy MJ. Differentiating solitary small cysticercosis granulomas and tuberculomas in patients with epilepsy; clinical and computed tomographic criteria. J Neurosurg $1993 ; 78 ; 402-7$

6. Kalra V. Central Nervous System. Ghai Essential Pediatrics. 7 th ed. New Delhi: CBS Publishers and Distributers Pvt Ltd, 2009:547.

7. Gupta RK, Pandey R, Khan EM, Mittal P, Gujral RB, Chhabra DK. Intracranial tubeculoma-MRI signal correlation with histopathology and localized proton spectroscopy. Magn Reson Imaging 1993;11:443-9.

8. Stephen M. Sagar, Mark A. In: Dennis L Kasper, Anthony S Fauci, Dan L Longo, Eugene Braunwald, Stephen L Hauser, J Larry Jameson (eds). Harrison's Principles of Internal Medicine. 16 ed. New York: Mc Graw Hill (Medical Publishing Division), 2005;2452.

9. Elisevich K, Aprin EJ. Tuberculoma masquerading as a meningioma. J of Neurosurg 1992: 56;435-8.
10. Bayindir C, Mete O, Bilgic B. Retrospective study of 23 pathologically proven cases of central nervous system tuberculoma. Clin Neurol Neurosurg 2006;108:353-7.

11. Mario CRavigilone, Richard J Obrien. In: Peter F Weller, Cestodes; Dennis L Kasper, Anthony S Fauci, Dan L Longo, Braunwald E, Stephen L Hauser, J larry Jameson, Joseph Loscalzo, et al (eds). Harrison's Principles of Internal Medicine. 17 eds. New York: Mc Graw Hill (Medical Publishing Division), 2008;1012.

12. Solomon T. In: Michael Donaghy ed. Brain's Disease of the Nervous System 12 ed. Oxford: Oxford university Press, 2009;1436-7.

13. Alexander J Mc Adam, Arlene H. In: Kumar V, Abul K Abbas, Fausto N, Jon C Aster (eds). Robbins and Cotran-Pathologic Basis of Disease. 8th eds. (South East Asian edition), Philadelphia: Saunders Elsevier, 2010:370. 